\title{
Originalarbeiten
}

\section{Chemisch-analytische Charakterisierung technischen tierischen Fettes aus einer Tierkörperbeseitigungsanstalt}

\author{
Müfit Bahadir ${ }^{1}$, Ralf Bock ${ }^{3}$, Tina Dettmer ${ }^{3}$, Oliver Falk ${ }^{2}$, Jürgen Hesselbach ${ }^{3}$, Petra Jopke', Birthe Matthies ${ }^{1}$, \\ Roland Meyer-Pittroff ${ }^{2}$, Christiane Schmidt-Nädler ${ }^{1}$, Hubertus Wichmann ${ }^{1 *}$ \\ ${ }^{1}$ Institut für Ökologische Chemie und Abfallanalytik, TU Braunschweig, Hagenring 30, D-38106 Braunschweig \\ ${ }^{2}$ Lehrstuhl für Energie- und Umwelttechnik der Lebensmittelindustrie, TU München, Weihenstephaner Steig 22, \\ D-85350 Freising-Weihenstephan \\ ${ }^{3}$ Institut für Werkzeugmaschinen und Fertigungstechnik, TU Braunschweig, Langer Kamp 19b, D-38106 Braunschweig
}

* Korrespondenzautor (hu.wichmann@tu-bs.de)

\section{DOI: http://dx.doi.org/10.1065/uwsf2003.03.054}

\section{Zusammenfassung}

Hintergrund und Ziel. Von den deutschen Tierkörperbeseitigungsanstalten werden $z$. Z. rund 300.000 t/a technisches tierisches Fett produziert. Ausgelöst durch die BSE-Krise wird dieser Rohstoff nicht mehr als Tierfutterergänzungsmittel eingesetzt. Nun wird nach neuen Verwertungswegen für diese Fette gesucht. In diesem Zusammenhang wird von der DBU ein Verbundprojekt finanziert, in dessen Rahmen durch Umesterung der Fette Grundöle für Kühlschmierstoffe (KSS) hergestellt und die KSS-Produkte in der spanenden Bearbeitung von Metallen in der industriellen Praxis getestet werden sollen.

Methoden. Als erster Schritt sollten durch chemisch-analytische Untersuchungen die Eigenschaften und die Qualität des Rohfettes festgestellt und im Verlauf eines Jahres verfolgt werden, um u. a. saisonal bedingte Schwankungen zu erkennen. Die fettspezifischen und spurenanalytischen Parameter umfassten Gesamtverschmutzung, Sulfatasche, Wassergehalt, Peroxidzahl, Iodzahl, kinematische Viskosität, Säurezahl (freie Fettsäuren) und Fettsäurespektrum, Elemente- bzw. Schwermetalle (Al, B, $\mathrm{Ca}, \mathrm{Cd}, \mathrm{Cr}, \mathrm{Cu}, \mathrm{Fe}, \mathrm{K}, \mathrm{Mo}, \mathrm{Mn}, \mathrm{Na}, \mathrm{Ni}, \mathrm{P}, \mathrm{Pb}, \mathrm{S}, \mathrm{Sn}, \mathrm{V}, \mathrm{W}$ und $\mathrm{Zn}$ ) sowie polycyclische aromatische Kohlenwasserstoffe, polychlorierte Biphenyle, polychlorierte Dibenzo-p-dioxine und polychlorierte Dibenzofurane.

Ergebnisse. Die fettspezifischen Parameter lieferten wertvolle Hinweise für die Planung und Auslegung des technischen Prozesses zur Herstellung von Fettsäuremethylestern. So erwiesen sich beispielsweise Filtration und Entwässerung der Fette als notwendig. Geringe Schwankungen von Iodzahl, Viskosität und Fettsäurespektrum deuteten auf eine recht konstante Zusammensetzung des Rohmaterials im Jahresverlauf hin. Der Anteil freier Fettsäuren lieferte Hinweise auf den Hydrolyse- und Verderbnisgrad des Fettes. Die für die Kühlschmierstoffherstellung interessierenden gesättigten Fettsäuren repräsentierten 36,4\% bis $54,6 \%$ des Fettsäurespektrums. Spurenanalytische Untersuchungen bezüglich anorganischer und organischer Schadstoffe belegten eine niedrige Grundbelastung des Rohfettes.

Ausblick. Ein nächster Schritt wird darin bestehen, die Technikumsanlage zur KSS-Grundölherstellung und damit verbunden Ergebnisse chemisch-analytischer Untersuchungen am Herstellungsprozess vorzustellen.

Schlagwörter: Fettanalytik; Kühlschmierstoff; PAK; PCB; PCDD/F; Schwermetall; Tierfett; Tierfettmethylester; Tierkörperbeseitigung

\section{Abstract}

Analytical Characterization of Technical Animal Fat from a Rendering Plant

Aims and Scope. German rendering plants produce 300,000 tons of animal fat per year. Until recently, these raw products have been in wide use as nutritional additive for cattle and other animals fodder but now they have been banned due to their assumed role in BSE infection. Therefore, alternative fields of usage are required. In this context the Deutsche Bundesstiftung Umwelt (DBU) is sponsoring a joint research project which deals with the production and testing of cooling lubricants based on animal fat esters.

Methods. In a first step, characteristics and quality of the animal fat were analyzed and monitored for a whole year in order to identify e.g. seasonal variations. The investigations covered the following fat specific and trace analytical parameters: total contamination, sulfates ash, water content, peroxide number, iodine value, kinematical viscosity, neutralisation number (free fatty acids), fatty acid spectra, elements / heavy metals ( $\mathrm{Al}, \mathrm{B}$, $\mathrm{Ca}, \mathrm{Cd}, \mathrm{Cr}, \mathrm{Cu}, \mathrm{Fe}, \mathrm{K}, \mathrm{Mo}, \mathrm{Mn}, \mathrm{Na}, \mathrm{Ni}, \mathrm{P}, \mathrm{Pb}, \mathrm{S}, \mathrm{Sn}, \mathrm{V}, \mathrm{W}$ and $\mathrm{Zn})$, polycyclic aromatic hydrocarbons, polychlorinated biphenyls, polychlorinated dibenzo-p-dioxins and polychlorinated dibenzofurans.

Results. Valuable hints on the design of the technical process of fatty acid methylesters production were gained by regarding the fat specific parameters. For example, filtration and dewatering of the fats proved necessary. Small variations of iodine value, viscosity and fatty acid spectrum pointed at a quite constant composition of the raw material in the course of the year. Clues to the degree of hydrolysis and decay of the fat were given by the share of free fatty acids. The saturated fatty acids, most interesting for the production of the cooling lubricants, were present in the fatty acid spectra in the rage of $36.4 \%$ to $54.6 \%$. Trace analytical investigations concerning inorganic and organic pollutants proved a low basic contamination of the technical animal fats.

Outlook. Next step will be to present the pilot plant for the production of the cooling lubricants and further analytical results in context with the production process.

Keywords: Animal fat; animal fat methylester; cooling lubricants; fat analysis; fatty acid spectrum; heavy metals; PAH, PCB; $\mathrm{CDD} / \mathrm{F}$; rendering plant 


\section{Einleitung}

Tierfett fällt gemeinsam mit Tiermehl bei der Tierkörperbeseitigung an. In Deutschland müssen jährlich 2.7 Mio. t Schlachtabfälle und Tierkörper in Tierkörperbeseitigungsanlagen behandelt werden, woraus $700000 \mathrm{t}$ Tiermehl und $300000 \mathrm{t}$ Tierfett resultieren. Bis vor kurzem wurden diese Abfallstoffe zu einem großen Teil als Tierfutterergänzungsmittel eingesetzt. Aufgrund der BSE-Problematik sollen sie jedoch nicht mehr zurück in die Nahrungskette gelangen. Dieser Verwertungsweg steht daher nicht mehr offen.

In einem von der Deutschen Bundesstiftung Umwelt geförderten Forschungsvorhaben soll eine alternative Verwertung von Tierfetten als Kühlschmierstoffe untersucht werden (DBU, Az. 17205, 'Kühlschmierstoffe aus Altspeisefetten und technischen tierischen Fetten').

Hierzu werden am Lehrstuhl für Energie- und Umwelttechnik der Lebensmittelindustrie, TU München-Weihenstephan, verschiedene Ester auf Basis von Tierfetten hergestellt, die anschließend am Institut für Werkzeugmaschinen und Fertigungstechnik, TU Braunschweig, auf ihre technologische Eignung im Zerspanungsprozess getestet werden. Hier wird auch in Zusammenarbeit mit der LCE Consulting $\mathrm{GmbH}$, Braunschweig, ein Life Cycle Assessment (Produktökobilanz) der neuen Produkte vorgenommen. Neben wirtschaftlichen, technologischen und ökologischen Aspekten, müssen auch arbeitsphysiologische Untersuchungen sowie eine chemisch-analytische Charakterisierung der Rohstoffe erfolgen. Diesen Teilbereich des Forschungsprojektes übernehmen das Institut für Ökologische Chemie und Abfallanalytik, TU Braunschweig und der Lehrstuhl für Energie- und Umwelttechnik der Lebensmittelindustrie, TU München-Weihenstephan.

Ziel der chemisch-analytischen Untersuchungen war es, Eigenschaften und Qualität des Rohmaterials, technisches tierisches Fett, festzustellen und im Verlauf eines Jahres durch wiederholte Probenahme aus einer deutschen Tierkörperbeseitigungsanstalt (TBA) zu verfolgen. Letzteres war erforderlich, um Parameterschwankungen, die u. a. saisonal bedingt sind, zu erfassen und Grundbelastungen mit Schadstoffen festzustellen. Die Messungen umfassten Gesamtverschmutzung, Sulfatasche, Wassergehalt, Peroxidzahl, lodzahl, kinematische Viskosität, Säurezahl (freie Fettsäuren) und Fettsäurespektrum, Element- bzw. Schwermetallgehalte (Al, B, Ca, Cd, Cr, Cu, Fe, K, Mo, Mn, Na, Ni, P, $\mathrm{Pb}, \mathrm{S}, \mathrm{Sn}, \mathrm{V}, \mathrm{W}$ und $\mathrm{Zn}$ ) sowie polyzyklische aromatische Kohlenwasserstoffe (PAK), polychlorierte Biphenyle (PCB), polychlorierte Dibenzo-p-dioxine (PCDD) und polychlorierte Dibenzofurane (PCDF), die in den letzten Jahren in der EU zu Futtermittelskandalen geführt hatten. Die resultierenden technisch wie toxikologisch interessanten Basisdaten werden nachfolgend vorgestellt und bewertet und damit der Fachöffentlichkeit zugänglich gemacht, die mit der Verwertung bzw. Entsorgung von technischem tierischem Fett befasst ist.

\section{Material und Methoden}

\subsection{Probenmaterial}

Die Fettproben (gereinigtes Tierfett) wurden aus der laufenden Produktion einer Tierkörperbeseitigungsanstalt entnommen. Abb. 1 zeigt den Produktionsprozess einer TBA und den Ort der Probenahme [1].

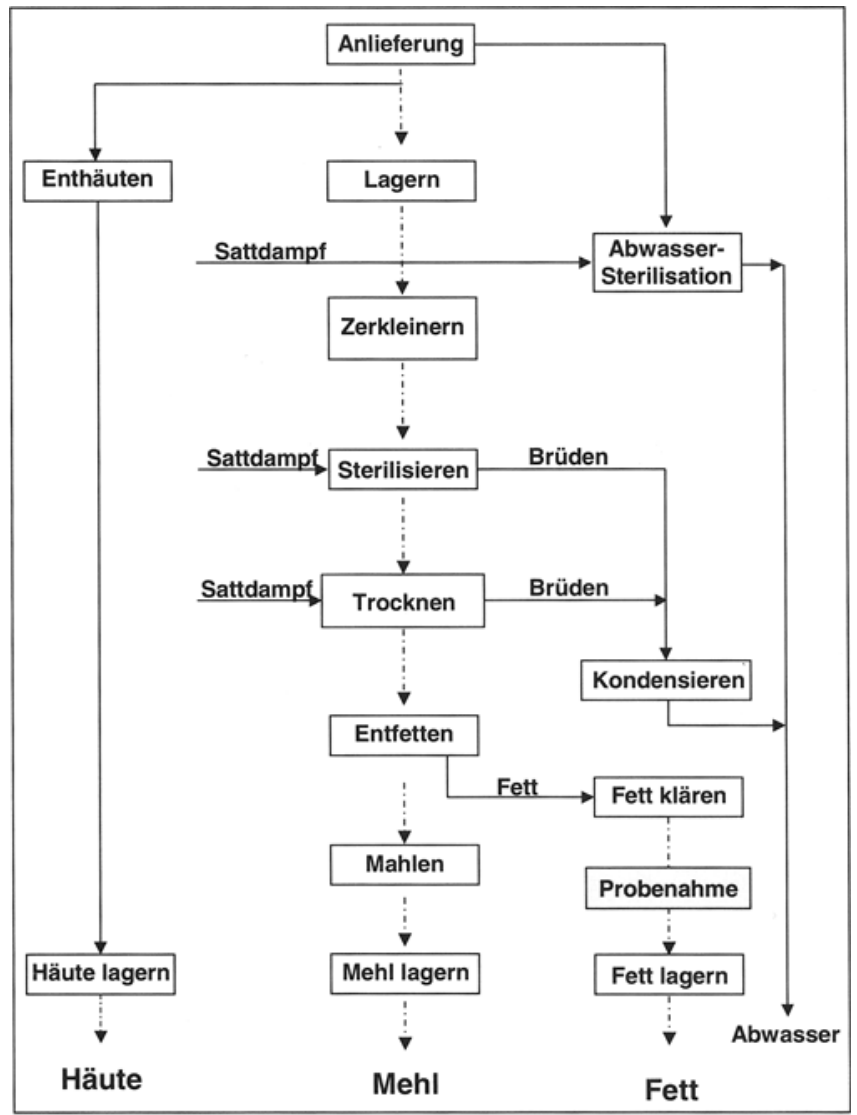

Abb. 1: Verfahrensablauf in einer Tierkörperbeseitigungsanlage

\subsection{Analytik fettspezifischer Parameter}

Um die technologische Eignung von Tierfetten für die Schmierstoffherstellung über den Jahresverlauf zu beurteilen, wurden die einzelnen Proben am Lehrstuhl für Energieund Umwelttechnik auf die in Tabelle 1 dargestellten Parameter untersucht.

Tabelle 1: Untersuchte fettspezifische Parameter der Tierfettproben und angewandte Methoden

\begin{tabular}{l|l}
\hline Prüfparameter & Prüfverfahren \\
\hline Gesamtverschmutzung & DIN 51419-A \\
\hline Sulfatasche & DIN 51575 \\
\hline Wassergehalt & ASTM D 1744 \\
\hline Peroxidzahl & $\begin{array}{l}\text { Nach Wheeler; DGF Einheitsmethoden: } \\
\text { C-VI 6a (97) }\end{array}$ \\
\hline lodzahl & DIN EN ISO 3961 \\
\hline Kin. Viskosität $\left(40^{\circ} \mathrm{C}\right)$ & DIN 51 562 \\
\hline Freie Fettsäuren & DGF Einheitsmethoden: C-V 2 (81) \\
\hline Fettsäurespektren & EN ISO 5508 \\
\hline
\end{tabular}

\subsection{Spurenanalytik}

Elementanalytik. Die Fettproben wurden einem mikrowelleninduzierten Druckaufschluss unterworfen (Aufschlussbedingungen: $0,5 \mathrm{~g}$ Probe, $8 \mathrm{~mL} \mathrm{HNO}_{3}, 65 \%$ ig und $2 \mathrm{~mL} \mathrm{H}_{2} \mathrm{O}_{2}$, $30 \%$ ig, Mikrowelle: $80^{\circ} \mathrm{C}, 43 \mathrm{psi}, 1 \mathrm{~min} ; 100^{\circ} \mathrm{C}, 87 \mathrm{psi}, 1 \mathrm{~min}$; $\left.120^{\circ} \mathrm{C}, 174 \mathrm{psi}, 1 \mathrm{~min} ; 180^{\circ} \mathrm{C}, 580 \mathrm{psi}, 15 \mathrm{~min}\right)$. Die Element- 
analytik erfolgte mittels optischer Emissionsspektrometrie mit induktiv gekoppeltem Plasma (ICP-OES).

Analytik der PAK. Die Fettproben wurden in Anlehnung an die DGF-Einheitsmethode C-III 17 a: Bestimmung von PAK in Ölen und Fetten [2] und das Untersuchungsverfahren nach $\$ 35$ LMBG 0700; 40: Bestimmung von Benzo[a]pyren in (geräucherten) Fleischerzeugnissen [3] untersucht.

Nach Verseifung des Fettes mit methanolischer KOH wurden die PAK (15 Leitsubstanzen in Anlehnung an US Environmental Protection Agency, ohne Naphthalin gemäß [2]) mit Cyclohexan extrahiert, in eine Dimethylformamid/Wasser-Mischung überführt und aus dieser erneut mit Cyclohexan extrahiert. Die Extrakte wurden unter Verwendung von Kieselgel Säulen (5 g Kieselgel, Wassergehalt 9,1\%, Elution mit $100 \mathrm{~mL}$ Cyclohexan) chromatographisch aufgereinigt. Die Quantifizierung der in der Probe enthaltenen PAK erfolgte nach der Methode des externen Standards mittels GC/MS. Als Einspritzstandard wurde Benzo[a]pyren- $\mathrm{d}_{12}$ eingesetzt.

Analytik der PCB. Jeweils $1 \mathrm{~g}$ der Fettproben wurde in $100 \mathrm{~mL}$ n-Hexan gelöst und durch Kochen unter Rückfluss über schwefelsaurem Kieselgel (30 g Kieselgel imprägniert mit 44\% konz. Schwefelsäure) aufgeschlossen. Die PCB wurden mittels einer Aluminiumoxid Säule ( $20 \mathrm{~g}$ neutrales Aluminiumoxid, Wassergehalt 2\%, Elution mit $60 \mathrm{~mL}$ n-Heptan/Essigsäureethylester (95:5)) von verbliebenen Begleitsubstanzen abgetrennt. Die Quantifizierung von sechs PCB-Leitsubstanzen erfolgte mit GC/MS-Meßtechnik und externer Kalibrierung.

Analytik der PCDD und PCDF. Die in Hexan gelösten Fettproben wurden analog zur PCB-Analytik durch Kochen unter Rückfluss über schwefelsaurem Kieselgel aufgeschlossen. Zuvor wurden der Lösung $50 \mu \mathrm{L}$ eines ${ }^{13} \mathrm{C}$-PCDD/F-Mischstandards $(50 \mathrm{pg} / \mu \mathrm{L})$ zugesetzt. Die PCDD/F wurden säulenchromatographisch durch Verwendung einer Kieselgel Säule weiter isoliert.

In eine Glassäule wurden nacheinander $2 \mathrm{~g}$ neutrales Kieselgel, $5 \mathrm{~g}$ basisches Kieselgel (mit $33 \% 1 \mathrm{~m} \mathrm{NaOH}$ ), 2 g neutrales Kieselgel, $10 \mathrm{~g}$ schwefelsaures Kieselgel (mit $44 \%$ $\mathrm{H}_{2} \mathrm{SO}_{4}$ konz.), 2 g neutrales Kieselgel sowie $10 \mathrm{~g} \mathrm{Na}_{2} \mathrm{SO}_{4}$ gegeben. Nach Probenaufgabe auf die Säule erfolgte die Elution mit $250 \mathrm{~mL}$ Heptan. Das Eluat wurde am Rotationsverdampfer auf ein Volumen von ca. $3 \mathrm{~mL}$ eingeengt und auf die nachfolgend beschriebene Alumina B Säule überführt.

In einer Glassäule wurden $25 \mathrm{~g}$ Alumina $\mathrm{B}$ mit $10 \mathrm{~g} \mathrm{Na}_{2} \mathrm{SO}_{4}$ überschichtet. Nach erfolgter Probenaufgabe wurde in einem ersten Elutionsschritt mit $100 \mathrm{~mL}$ einer Heptan/Essigsäureethylester-Lösung (99:1) eluiert; das Eluat wurde verworfen. Im zweiten Schritt wurde mit $180 \mathrm{~mL}$ einer Mischung Heptan/Essigsäureethylester (9:1) eluiert. Das Eluat wurde bis zur Trockene eingeengt. Der Rückstand wurde in $50 \mu \mathrm{L}$ i-Octan sowie $50 \mu \mathrm{L}$ eines ${ }^{13} \mathrm{C}-1,2,3,4-T e C D D$ Standards $(50 \mathrm{pg} / \mu \mathrm{L}$ ) aufgenommen. Die Bestimmung der PCDD/PCDF erfolgte mit GC/MS unter Anwendung der Isotopenverdünnungsmethode.

\section{Ergebnisse und Diskussion}

\subsection{Analysenergebnisse fettspezifischer Parameter}

In den Abb. 2 bis 9 sind die Analysenergebnisse der fettspezifischen Parameter dargestellt.

Wie aus den Abb. 2 und 3 ersichtlich ist, bewegen sich die Verschmutzungsraten der Tierfette auf stark schwankendem hohen Niveau. Bei Gesamtverschmutzungsraten von bis zu $1,4 \%$ müssen die Fette vor der Weiterverarbeitung auf jeden Fall filtriert werden.

Die Sulfataschewerte korrelieren nicht mit den Werten der Gesamtverschmutzung, was darauf hinweist, dass ein Teil der erfassten Gesamtverschmutzung organischen Ursprungs ist.

Die Wassergehalte (Abb. 4) bewegen sich, abgesehen von einem Ausreißer auf einem durchgehend niedrigen Niveau. Für die weitere Verarbeitung empfiehlt sich dennoch eine zusätzliche Entwässerung durch Destillation, da es sonst zu Störungen der Umesterungsreaktion durch Katalysatorverlust kommen kann.

Bei der Peroxidzahl (Abb. 5) waren aufgrund der starken oxidativen Belastung des Rohmaterials durchgehend sehr hohe Werte erwartet worden. Das sehr weite Spektrum der

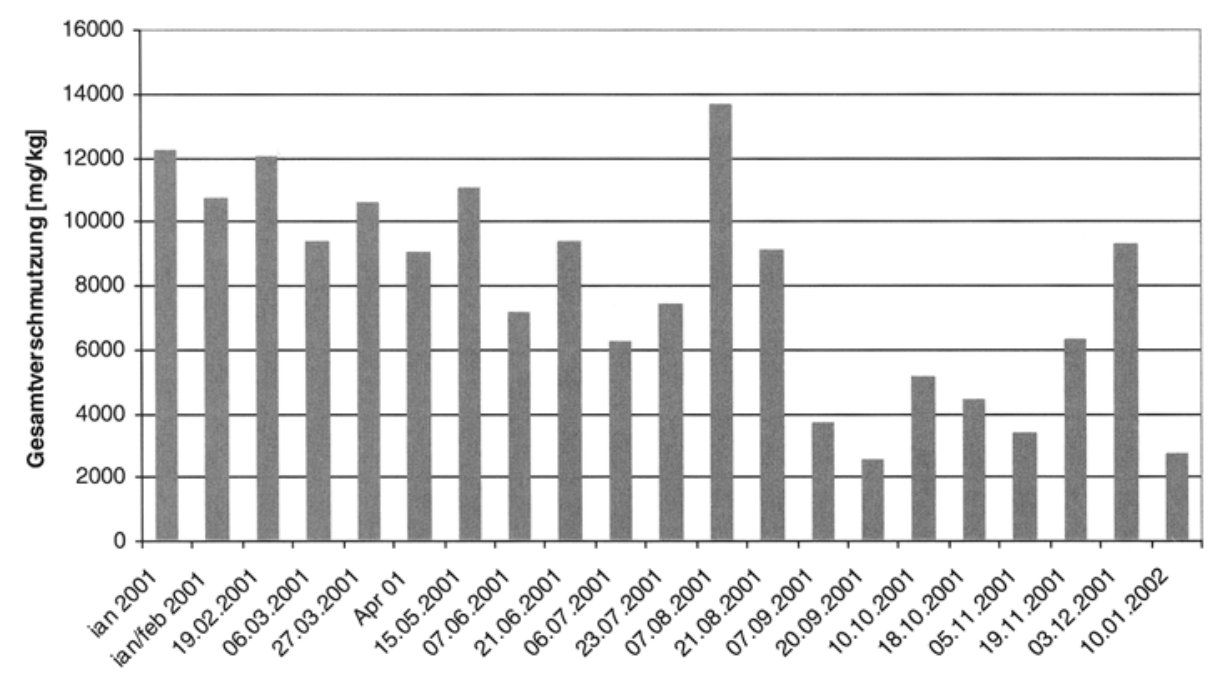

Abb. 2: Gesamtverschmutzung der Rohfettproben 


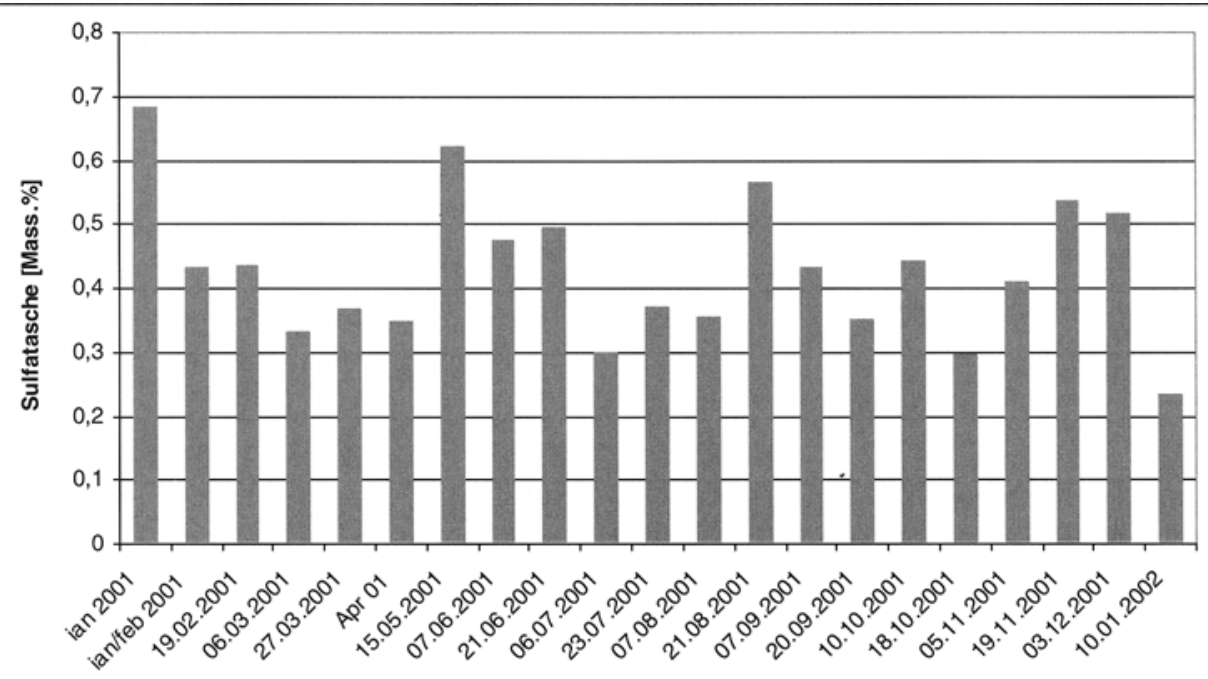

Abb. 3: Sulfatasche der Rohfettproben

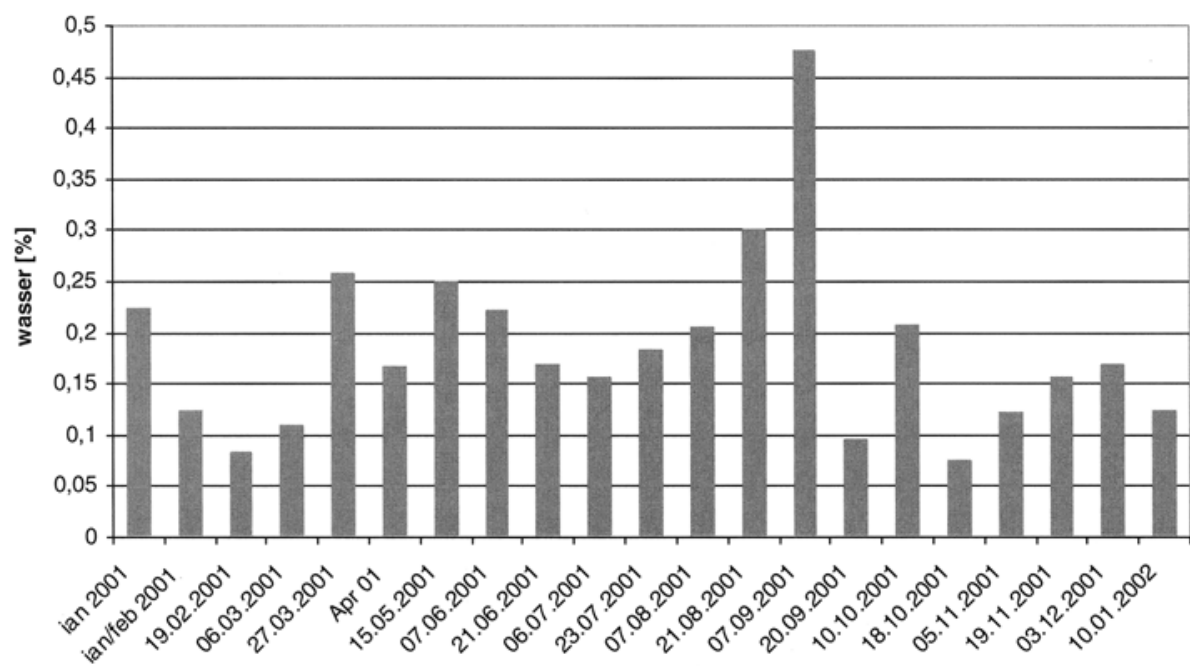

Abb. 4: Wassergehalt der Rohfettproben

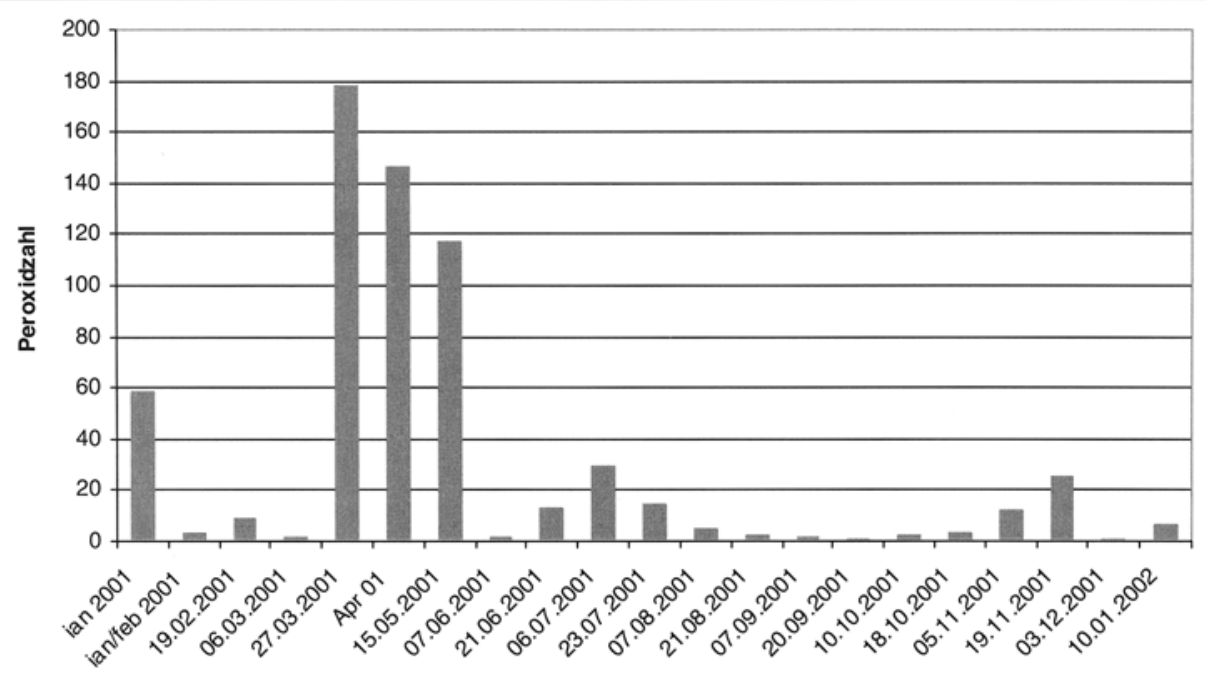

Abb. 5: Peroxidzahl der Rohfettproben 


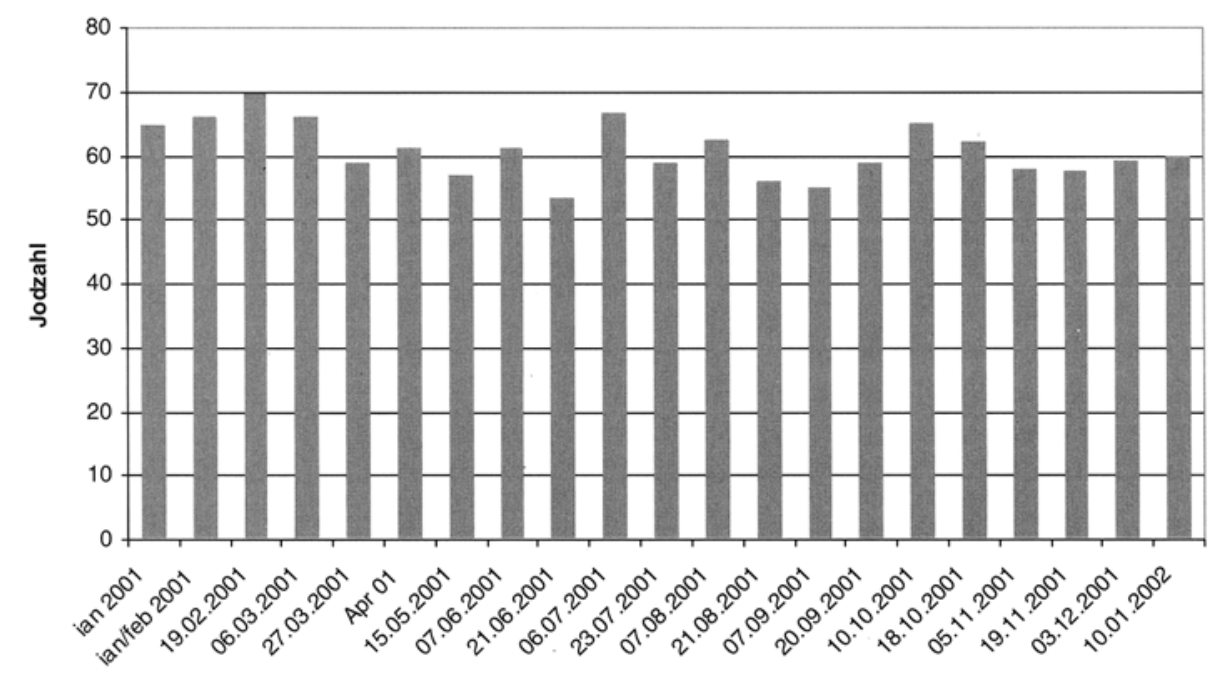

Abb. 6: lodzahl der Rohfettproben

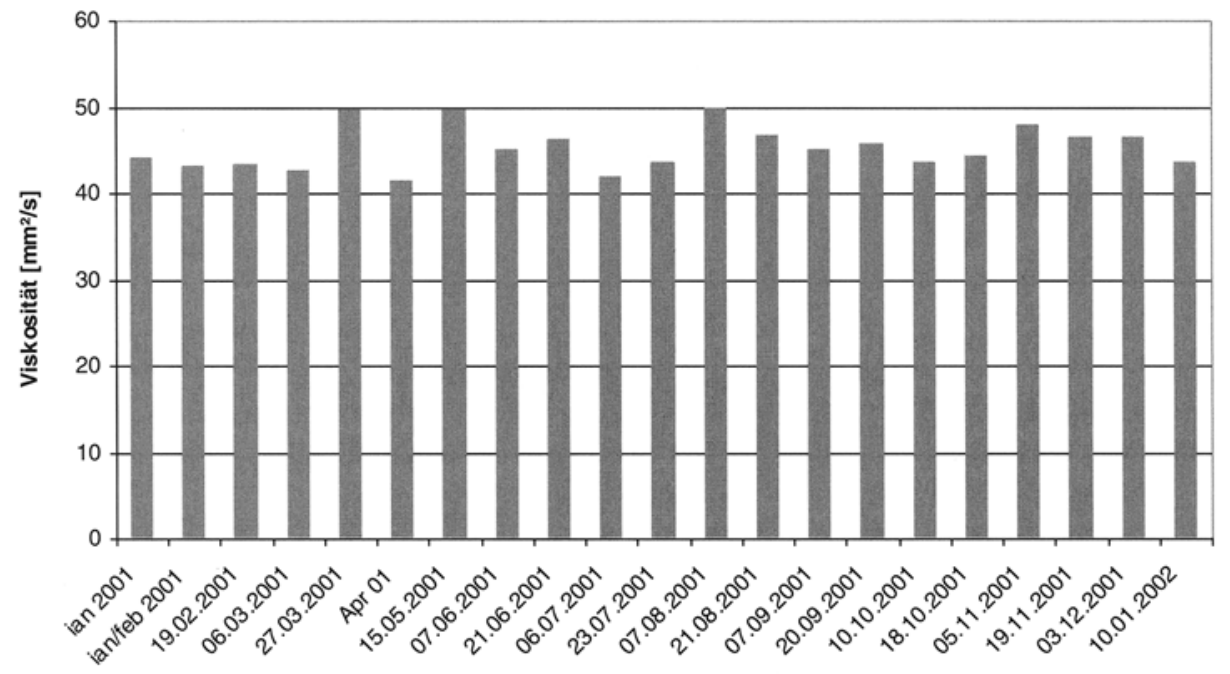

Abb. 7: Kinematische Viskosität der Rohfettproben

POZ von 0,5 bis fast 180 lässt sich dadurch erklären, dass eine Vielzahl der Proben so stark verdorben war, dass sich gebildete Hydroperoxide bereits wieder zersetzt hatten. Die Peroxidzahl scheint daher keine geeignete Analysenmethode zur Beurteilung des Verderbnisgrades der Tierfette zu sein.

Die Iodzahlen (Abb. 6) und die kinematischen Viskositäten (Abb. 7) weisen über den Jahresverlauf nur sehr geringe Schwankungen auf. Dies liegt vor allem an der gleichmäßigen Zusammensetzung des verarbeiteten Rohmaterials (v.a. Schwein und Rind, vgl. Abb. 9: Fettsäurespektren). Die leichten Schwankungen bei der Viskosität können durch einen unterschiedlichen Hydrolyse- und Polymerisationsgrad der einzelnen Proben erklärt werden.

Während man bei frischem Schweine- bzw. Rinderfett mit freien Fettsäuren unter 1\% rechnet, erhält man bei den untersuchten Proben Werte bis über 22\%. Die freien Fettsäu- ren (berechnet aus der Säurezahl; Abb. 8) können somit als Indikator für den Hydrolyse- und Verderbnisgrad des Rohmaterials angesehen werden. Über den Jahresverlauf kann man in den Sommermonaten höhere FFA-Gehalte beobachten. Dies lässt sich durch einen stärkeren mikrobiologischen Verderb, bedingt durch die höheren Temperaturen während der Lagerung, erklären.

Die Proben weisen über den Jahresverlauf nur geringe Schwankungen in der Fettsäurezusammensetzung auf. Die prozentual größten Unterschiede bemerkt man bei der Stearinsäure (18:0) und der Linolsäure (18:2). Geringe Mengen an Stearinsäure können darauf hinweisen, dass ein größerer Anteil Geflügel verarbeitet wurde (Abb. 9).

Der Anteil an den für die Weiterverarbeitung zu Schmierstoffen besonders interessanten gesättigten Fettsäuren bewegt sich zwischen $36,4 \%$ und $54,6 \%$. 

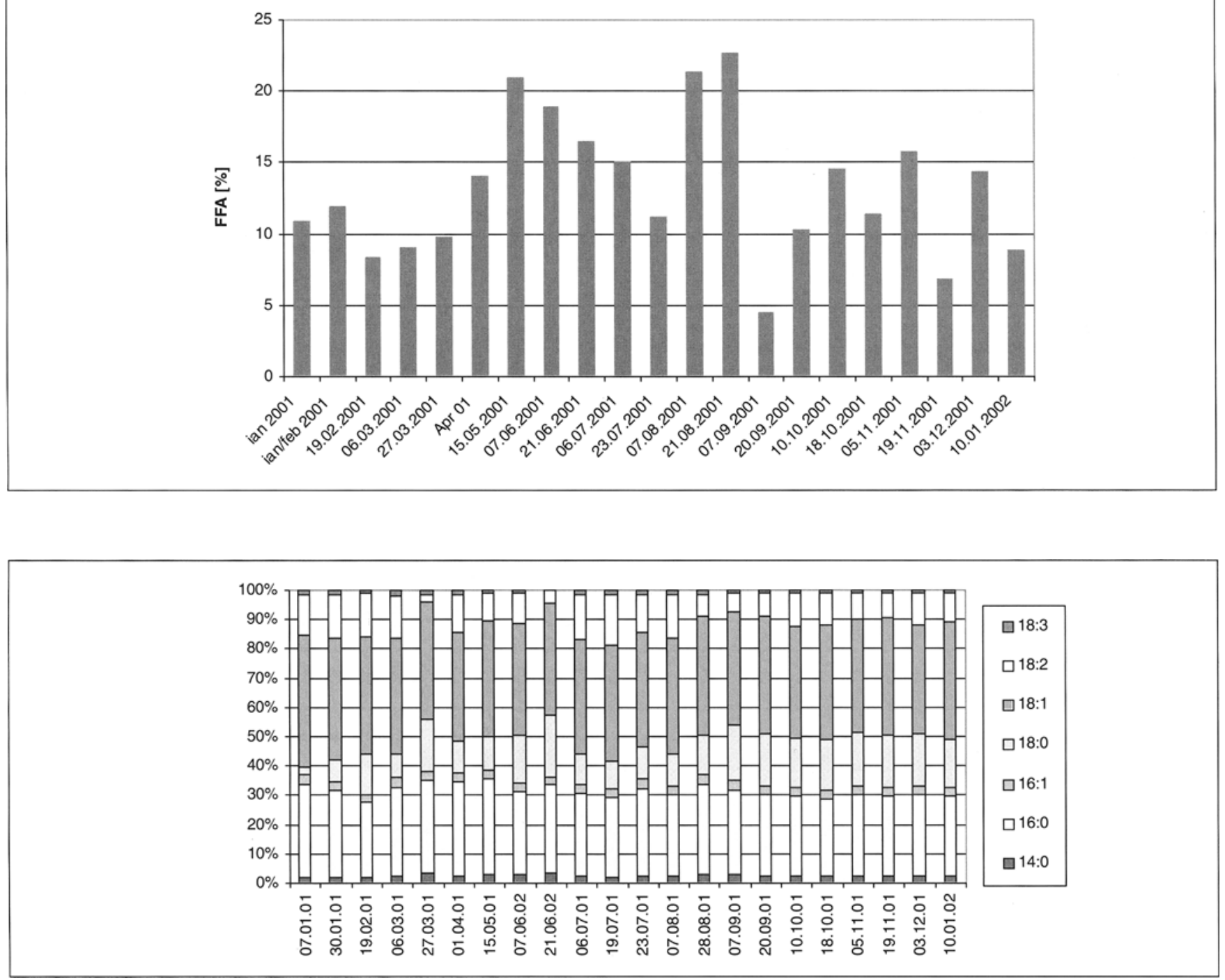

\subsection{Ergebnisse der Spurenanalytik}

Elementgehalte. Die Ergebnisse der Elementanalytik sind in den Tabellen 2 und 3 aufgeführt.

Bei Betrachtung der in den Tabellen 2 und 3 gelisteten Werte sind keine jahreszeitlich bedingten Konzentrationsschwankungen festzustellen. Korrelationen sind lediglich bei den Konzentrationen der Elemente $\mathrm{Ca}$ und $\mathrm{P}$ sowie $\mathrm{Na}$ und $\mathrm{K}$ erkennbar.

Die bisher in der Literatur verfügbaren Angaben zu Schwermetallgehalten von technischen tierischen Fetten gehen auf eine Information des Bayerischen Landesamtes für Umweltschutz aus dem Jahre 2001 zurück. Folgende Werte wurden dort angegeben [4]:

$\begin{array}{lrl}\text { Cd: } & <50 & \text { ng/g Fett } \\ \text { Cr: } & 300 & \text { ng/g Fett } \\ \text { Cu: } & 500 & \text { ng/g Fett } \\ \text { Ni: } & <100 & \text { ng/g Fett } \\ \text { Pb: } & 400 & \text { ng/g Fett }\end{array}$

Die Angaben konnten durch die im Rahmen dieser Untersuchung festgestellten Konzentrationen, die ca. um den Faktor 100 niedriger waren, nicht bestätigt werden.

In Tabelle 4 sind die Analysendaten aus der Elementanalytik in der Weise aufbereitet, dass jeweils der Konzentrationsbereich und der Jahresmittelwert der Konzentrationen angegeben werden. Außerdem sind die Elemente nach der Höhe der mittleren Konzentrationen sortiert. Bei Unterschreitung der Bestimmungsgrenze wurde, wie üblich, der halbe Wert dieses analysentechnisch bedingten Limits in die Rechnung einbezogen. Bei Unterschreitung der jeweiligen Bestimmungsgrenze bei mehr als sechs Proben wird kein Jahresmittelwert angegeben.

Wie aufgrund der Art der Proben zu erwarten war, dominieren innerhalb der Gruppe der untersuchten Elemente von ihrer Konzentration her $\mathrm{Ca}, \mathrm{P}, \mathrm{Na}, \mathrm{K}$ und $\mathrm{S}$. Erst danach ist das toxikologisch unbedeutende Schwermetall Fe sowie das Element B einzuordnen. Die toxikologisch potentiell relevanten Schwermetalle $\mathrm{Ni}, \mathrm{Cu}, \mathrm{Zn}$ und $\mathrm{Cr}$ sowie das Leichtmetall Al konnten im Mittel im Konzentrationsbereich zwi- 
Tabelle 2: Elementgehalte in den Rohfettproben Januar 2001 bis Juni 2001 in [ng/g Fett]

\begin{tabular}{|c|c|c|c|c|c|c|}
\hline Element & Januar 01 & Februar 01 & März 01 & April 01 & Mai 01 & Juni 01 \\
\hline Al & 6,03 & 7,00 & 3,86 & 7,74 & $<2,5$ & 5,34 \\
\hline B & 4,68 & 13,7 & 4,03 & 2,66 & 1,87 & 8,96 \\
\hline $\mathrm{Ca}$ & 1.340 & 863 & 503 & 73,7 & 77,5 & 223 \\
\hline $\mathrm{Cd}$ & $<2,5$ & $<2,5$ & $<2,5$ & $<2,5$ & $<2,5$ & $<2,5$ \\
\hline $\mathrm{Cr}$ & 4,73 & 5,48 & 4,57 & 5,46 & 4,46 & 4,41 \\
\hline $\mathrm{Cu}$ & 8,59 & 2,83 & 4,62 & 8,96 & 13,3 & 11,6 \\
\hline $\mathrm{Fe}$ & 35,6 & 34,3 & 25,9 & 27,3 & 27,6 & 29,2 \\
\hline K & 109 & 69,5 & 38,1 & 135 & 357 & 291 \\
\hline Mo & 1,31 & 2,76 & 1,38 & 2,97 & 2,55 & 2,27 \\
\hline $\mathrm{Mn}$ & 1,53 & 1,58 & 1,37 & 1,35 & 1,34 & 1,38 \\
\hline $\mathrm{Na}$ & 209 & 131 & 133 & 185 & 339 & 255 \\
\hline $\mathrm{Ni}$ & 9,13 & 9,08 & 9,34 & 9,72 & 8,71 & 9,13 \\
\hline $\mathbf{P}$ & 940 & 556 & 276 & 84,6 & 107 & 239 \\
\hline $\mathrm{Pb}$ & $<2,5$ & $<2,5$ & 2,79 & 3,26 & $<2,5$ & $<2,5$ \\
\hline $\mathrm{s}$ & 95,5 & 116 & 81,4 & 182 & 104 & 91,5 \\
\hline Sn & $<2,5$ & $<2,5$ & 4,72 & 3,03 & $<2,5$ & 5,15 \\
\hline v & $<2,5$ & $<2,5$ & $<2,5$ & $<2,5$ & $<2,5$ & $<2,5$ \\
\hline w & $<2,5$ & $<2,5$ & $<2,5$ & $<2,5$ & $<2,5$ & $<2,5$ \\
\hline $\mathrm{Zn}$ & 7,21 & 3,87 & 4,14 & 6,42 & 5,52 & 5,82 \\
\hline
\end{tabular}

Tabelle 3: Elementgehalte in den Rohfettproben Juli 2001 bis Dezember 2001 in [ng/g Fett]

\begin{tabular}{|c|c|c|c|c|c|c|}
\hline Element & Juli 01 & August 01 & September 01 & Oktober 01 & November 01 & Dezember 01 \\
\hline Al & 14,3 & 5,81 & 12,0 & 11,3 & 10,9 & 7,61 \\
\hline B & 14,8 & 12,3 & 19,5 & 25,3 & 3,97 & 14,7 \\
\hline $\mathrm{Ca}$ & 1.580 & 143 & 726 & 606 & 925 & 387 \\
\hline Cd & $<2,5$ & $<2,5$ & $<2,5$ & $<2,5$ & $<2,5$ & $<2,5$ \\
\hline $\mathrm{Cr}$ & 3,97 & 5,03 & 4,97 & 4,31 & $<2,5$ & $<2,5$ \\
\hline $\mathrm{Cu}$ & 7,71 & 7,32 & $<2,5$ & 4,07 & $<2,5$ & $<2,5$ \\
\hline $\mathrm{Fe}$ & 39,9 & 37,3 & 31,1 & 34,4 & 7,10 & 5,42 \\
\hline $\mathbf{K}$ & 162 & 361 & 116 & 156 & 43,2 & 149 \\
\hline Mo & 1,51 & 2,24 & 2,16 & 2,20 & $<1,0$ & $<1,0$ \\
\hline Mn & 1,30 & 1,41 & 1,41 & 1,42 & $<1,0$ & $<1,0$ \\
\hline $\mathrm{Na}$ & 301 & 873 & 145 & 249 & 189 & 191 \\
\hline $\mathrm{Ni}$ & 8,18 & 9,26 & 9,35 & 9,31 & $<2,5$ & $<2,5$ \\
\hline $\mathbf{P}$ & 1.430 & 133 & 422 & 392 & 545 & 327 \\
\hline $\mathrm{Pb}$ & 5,04 & $<2,5$ & $<2,5$ & $<2,5$ & $<2,5$ & $<2,5$ \\
\hline $\mathrm{s}$ & 116 & 176 & 151 & 111 & 46,3 & 83,3 \\
\hline Sn & 3,68 & $<2,5$ & $<2,5$ & $<2,5$ & $<2,5$ & 11,6 \\
\hline v & $<2,5$ & $<2,5$ & $<2,5$ & $<2,5$ & $<2,5$ & $<2,5$ \\
\hline W & $<2,5$ & $<2,5$ & $<2,5$ & $<2,5$ & $<2,5$ & $<2,5$ \\
\hline $\mathrm{Zn}$ & 7,04 & 5,75 & 1,99 & 4,62 & 4,33 & 2,78 \\
\hline
\end{tabular}


Tabelle 4: Konzentrationsbereiche und mittlere Konzentrationen der Elemente in den Rohfettproben in [ng/g Fett]

\begin{tabular}{|c|c|c|}
\hline Element & Konzentrationsbereich & Jahresmittelwert \\
\hline $\mathrm{Ca}$ & $73,7-1.580$ & 621 \\
\hline $\mathbf{P}$ & $84,6-1.430$ & 454 \\
\hline $\mathrm{Na}$ & $131-873$ & 267 \\
\hline $\mathbf{K}$ & $38,1-357$ & 166 \\
\hline $\mathbf{S}$ & $46,3-182$ & 113 \\
\hline $\mathrm{Fe}$ & $5,42-39,9$ & 27,9 \\
\hline B & $1,87-25,3$ & 10,5 \\
\hline $\mathrm{Ni}$ & $<2,5-9,72$ & 7,81 \\
\hline Al & $<2,5-14,3$ & 7,76 \\
\hline $\mathrm{Cu}$ & $<2,5-13,3$ & 6,02 \\
\hline $\mathrm{Zn}$ & $1,99-7,21$ & 4,96 \\
\hline $\mathrm{Cr}$ & $<2,5-5,48$ & 4,16 \\
\hline Mo & $<1,0-2,97$ & 1,86 \\
\hline $\mathrm{Mn}$ & $<1,0-1,58$ & 1,26 \\
\hline Sn & $<2,5-11,6$ & n.b. ${ }^{a}$ \\
\hline $\mathrm{Pb}$ & $<2,5-5,04$ & n.b. ${ }^{a}$ \\
\hline Cd & $<2,5$ & n.b. ${ }^{a}$ \\
\hline V & $<2,5$ & n.b. ${ }^{a}$ \\
\hline W & $<2,5$ & n.b. ${ }^{a}$ \\
\hline \multicolumn{3}{|c|}{${ }^{a}$ n.b.: nicht bestimmt } \\
\hline
\end{tabular}

schen 4 und $8 \mathrm{ng} / \mathrm{g}$ Rohfett nachgewiesen werden. Die Schwermetalle Mo, Mn, Sn, Pb, Cd, V und W waren in den untersuchten Fettproben zumeist sehr niedrig konzentriert und damit von untergeordneter Bedeutung.

PAK-Gehalte. In den Tabellen 5 und 6 sind die Gehalte der PAK-Leitsubstanzen in den Fettproben dargestellt. Die Konzentrationen von Benzo[b]fluoranthen und Benzo[k]fluoranthen werden als Summe angegeben, da die Substanzen gaschromatographisch nicht vollständig getrennt wurden:

Die Konzentrationssummen der 15 untersuchten PAK Leitsubstanzen liegen zwischen 25,0 und $121 \mathrm{ng} / \mathrm{g}$ Fett. Phenanthren ist mit Konzentrationen zwischen 6,84 und 46,5 ng/g Fett die dominierende Einzelsubstanz. Ein Trend im jahreszeitlichen Verlauf der Konzentrationen ist nicht feststellbar. Die DGF teilte in einer Pressemitteilung aus dem Jahr 2001 [5] mit, dass native Speiseöle Gehalte von 30 bis $120 \mathrm{ng}$ PAK/g Öl aufweisen können. Für Benzo[a]pyren wurde ein Grenzwert für Speiseöle von $5 \mathrm{ng} / \mathrm{g}$ vorgeschlagen. Ein Vergleich der Werte in den Tabellen 4 und 5 mit Angaben aus dem Lebensmittelsektor verdeutlicht die Unbedenklichkeit der Grundbelastung des Rohfettes mit PAK. Allerdings ist weiterzuverfolgen, in welchem Ausmaß PAK bei thermischer

Tabelle 5: PAK-Gehalte in den Rohfettproben Januar 2001 bis Juni 2001 in [ng/g Fett]

\begin{tabular}{l|c|c|c|c|c|c}
\hline PAK & Januar 01 & Februar 01 & März 01 & April 01 & Mai 01 & Juni 01 \\
\hline Acenaphthylen & 6,57 & 8,96 & 5,98 & 2,77 & 1,52 & $<0,5$ \\
\hline Acenaphthen & 2,22 & 3,31 & 1,99 & 2,57 & 0,85 & $<0,5$ \\
\hline Fluoren & 9,91 & 12,9 & 7,58 & 3,86 & 3,37 & 1,32 \\
\hline Phenanthren & 40,0 & 46,5 & 29,9 & 9,37 & 12,6 & 6,84 \\
\hline Anthracen & 7,43 & 9,53 & 6,36 & 3,37 & 2,15 & 0,78 \\
\hline Fluoranthen & 11,3 & 11,9 & 9,15 & 5,78 & 4,72 & 3,81 \\
\hline Pyren & 11,9 & 11,4 & 9,52 & 6,61 & 5,44 & 4,28 \\
\hline Benz[a]anthracen & 2,96 & 2,87 & 2,32 & 3,88 & 1,59 & 1,25 \\
\hline Chrysen & 3,92 & 3,52 & 3,13 & 4,66 & 2,53 & 1,82 \\
\hline Benzo[b]+[k]fluoranthen & 4,07 & 3,66 & 3,06 & 7,31 & 2,30 & 3,63 \\
\hline Benzo[a]pyren & 1,94 & 1,74 & 1,26 & 3,70 & 1,37 & 1,05 \\
\hline Indeno[1,2,3,c,d]pyren & 1,92 & 1,45 & 1,21 & 3,63 & 1,54 & 1,12 \\
\hline Dibenz[a,h]anthracen & 1,11 & 0,59 & $<0,5$ & 3,25 & 0,69 & $<0,5$ \\
\hline Benzo[g,h,i]perylen & 2,63 & 2,26 & 1,72 & 3,88 & 1,56 & 1,30 \\
\hline Summe PAK & $\mathbf{1 0 8}$ & $\mathbf{1 2 1}$ & $\mathbf{8 3 , 7}$ & $\mathbf{6 4 , 6}$ & $\mathbf{4 2 , 2}$ & $\mathbf{2 8 , 5}$ \\
\hline
\end{tabular}

Tabelle 6: PAK-Gehalte in den Rohfettproben Juli 2001 bis Dezember 2001 in [ng/g Fett]

\begin{tabular}{l|c|c|c|c|c|c}
\hline PAK & Juli $\mathbf{0 1}$ & August $\mathbf{0 1}$ & September 01 & Oktober 01 & November 01 & Dezember 01 \\
\hline Acenaphthylen & 0,65 & 0,82 & $\mathbf{1 , 7 8}$ & $\mathbf{1 , 2 0}$ & 1,86 & 0,75 \\
\hline Acenaphthen & $<0,5$ & 0,97 & 1,98 & 1,02 & 1,24 & 0,99 \\
\hline Fluoren & $\mathbf{1 , 7 8}$ & 4,89 & 4,30 & 3,13 & 6,36 & 3,58 \\
\hline Phenanthren & 8,10 & 24,9 & 13,7 & 12,4 & 26,2 & 12,8 \\
\hline Anthracen & 1,11 & 2,01 & 2,71 & 1,57 & 4,98 & 1,26 \\
\hline Fluoranthen & 2,58 & 21,3 & 5,85 & 5,68 & 8,39 & 7,79 \\
\hline Pyren & 3,21 & 16,5 & 6,97 & 6,63 & 8,40 & 12,9 \\
\hline Benz[a]anthracen & 0,95 & 3,05 & 2,14 & 1,68 & 2,25 & 1,40 \\
\hline Chrysen & 1,33 & 2,63 & 2,81 & 2,45 & 3,15 & 2,11 \\
\hline Benzo[b]+[k]fluoranthen & 1,67 & 6,32 & 5,09 & 3,83 & 6,00 & 3,18 \\
\hline Benzo[a]pyren & 1,20 & 1,82 & 5,01 & 2,44 & 2,17 & 1,13 \\
\hline Indeno[1,2,3,c,d]pyren & 0,75 & 1,41 & 1,68 & 1,52 & 1,59 & 0,89 \\
\hline Dibenz[a,h]anthracen & $<0,5$ & 0,53 & 1,09 & $<0,5$ & $<0,5$ & $<0,5$ \\
\hline Benzo[g,h,i]perylen & 0,89 & $\mathbf{1 , 7 0}$ & 2,18 & 1,91 & 2,09 & 1,14 \\
\hline Summe PAK & $\mathbf{2 5 , 0}$ & $\mathbf{8 8 , 8}$ & $\mathbf{5 7 , 3}$ & $\mathbf{4 5 , 8}$ & $\mathbf{7 5 , 0}$ & $\mathbf{5 0 , 1}$ \\
\hline
\end{tabular}


Tabelle 7: PCB-Gehalte in den Rohfettproben Januar 2001 bis Juni 2001 in [ng/g Fett]

\begin{tabular}{|c|c|c|c|c|c|c|}
\hline PCB & Januar 01 & Februar 01 & März 01 & April 01 & Mai 01 & Juni 01 \\
\hline PCB 28 & $<2,0$ & $<2,0$ & $<2,0$ & 9,06 & 6,68 & 4,16 \\
\hline PCB 52 & $<2,0$ & $<2,0$ & $<2,0$ & $<2,0$ & 4,14 & $<2,0$ \\
\hline PCB 101 & 3,66 & 4,00 & 5,04 & 9,46 & 6,18 & 6,56 \\
\hline PCB 138 & 13,8 & 12,2 & 11,9 & 16,6 & 9,82 & 9,54 \\
\hline PCB 153 & 20,2 & 16,0 & 18,9 & 19,5 & 11,5 & 12,3 \\
\hline PCB 180 & 9,22 & 7,86 & 9,04 & 19,4 & 8,88 & 10,0 \\
\hline Summe PCB & 47,1 & 40,1 & 44,9 & 74,1 & 47,2 & 42,5 \\
\hline
\end{tabular}

Tabelle 8: PCB-Gehalte in den Rohfettproben Juli 2001 bis Dezember 2001 in [ng/g Fett]

\begin{tabular}{|c|c|c|c|c|c|c|}
\hline PCB & Juli 01 & August 01 & September01 & Oktober 01 & November 01 & Dezember 01 \\
\hline PCB 28 & $<2,0$ & $<2,0$ & 2,02 & 2,08 & $<2,0$ & $<2,0$ \\
\hline PCB 52 & $<2,0$ & $<2,0$ & $<2,0$ & $<2,0$ & 5,92 & $<2,0$ \\
\hline PCB 101 & 5,30 & 5,70 & 5,10 & 3,62 & 3,28 & 3,32 \\
\hline PCB 138 & 10,2 & 10,5 & 8,62 & 8,28 & 7,70 & 8,84 \\
\hline PCB 153 & 10,9 & 13,3 & 10,9 & 9,24 & 11,2 & 12,6 \\
\hline PCB 180 & 8,46 & 11,0 & 7,94 & 7,10 & 6,64 & 6,68 \\
\hline Summe PCB & 34,9 & 40,6 & 34,5 & 30,2 & 34,7 & 33,3 \\
\hline
\end{tabular}

Belastung während dér geplanten Weiterverarbeitung des Fettes und des Einsatzes der späteren Produkte gebildet werden.

PCB-Gehalte. Die Tabellen 7 und 8 enthalten die Konzentrationen der sechs PCB-Leitsubstanzen in den Proben des technischen tierischen Fettes. Nachfolgend werden für die Substanzen diese Kurzbezeichnungen verwendet:

2,4,4'-Trichlorbiphenyl: PCB 28; 2,2' ${ }^{\prime} 5,5^{\prime}$-Tetrachlorbiphenyl: PCB 52; 2,2',4,5,5'-Pentachlorbiphenyl: PCB 101; $2,2^{\prime}, 3,4,4^{\prime}, 5^{\prime}$-Hexachlorbiphenyl: PCB 138; 2,2', 4, $4^{\prime}, 5,5^{\prime}-$ Hexachlorbiphenyl: PCB 153; 2, $2^{\prime}, 3,4,4^{\prime}, 5,5^{\prime}$-Heptachlorbiphenyl: PCB 180.

Bei Betrachtung der Tabellen 7 und 8 fällt auf, dass die höherchlorierten Kongenere PCB 138, РCB 153 und PCB 180 in höheren Konzentrationen nachweisbar waren, als die niedriger chlorierten. Diese Feststellung stimmt mit Ergebnissen aus Fütterungsversuchen mit PCB-kontaminiertem Futter überein [6]. Dort wurde eine enge Beziehung zwischen den PCB-Konzentrationen im Futter und.im Fettgewebe der Tiere für die höherchlorierten PCB gefunden. In der Verordnung über Höchstmengen von Schadstoffen in Lebensmitteln $(\mathrm{SHmV})$ [7] werden folgende PCB-Grenzwerte für tierische Speisefette außer Milchfett genannt:

Konzentrationssumme

PCB 28, PCB 52, PCB 101 und PCB 180: 80 ng/g Fett

Konzentrationssumme

PCB 138 und PCB 153:

100 ng/g Fett

Wie bei den PAK wird auch hier durch einen Vergleich mit Angaben aus dem Lebensmittelbereich die Unbedenklichkeit der PCB-Grundbelastung dieses Fettes belegt, da die Summen der Konzentrationen der sechs PCB Leitsubstanzen zwischen 30,2 und 74,1 ng/g Fett liegen und somit selbst in der Form die o.g. Grenzwerte der SHmV unterschritten werden.
PCDD/PCDF-Gehalte. In den 12 untersuchten Proben der technischen tierischen Fette wurden keine PCDD- und PCDFKongenere oberhalb der Bestimmungsgrenze von $0,1 \mathrm{ng} / \mathrm{g}$ Fett nachgewiesen. Gemäß Gefahrstoffverordnung [8] ist eine Zubereitung mit allen arbeitsrechtlichen Konsequenzen erst als krebserregend anzusehen, wenn die PCDD/F-Konzentration mindestens $2 \mathrm{ng}$ TE/g Fett beträgt. Unter der Annahme, dass alle 17 toxikologisch relevanten PCDD- und PCDFKongenere mit der Konzentration der Bestimmungsgrenze vorlägen, ergäbe sich ein Gehalt von 0,169 ng (WHO)TE/g Fett. Die Analysenergebnisse für PCDD und PCDF zeigen somit die Unbedenklichkeit des Rohfettes in Bezug auf diese Schadstoffgruppen, wenn lediglich eine sehr niedrige Grundbelastung vorliegt.

\subsection{Bewertung}

Die fettspezifischen Analysenparameter sind vor allem für den technologischen Prozess der Schmierstoffherstellung von Bedeutung. So stellte sich zum Beispiel heraus, dass die hohe Gesamtverschmutzung verbunden mit dem hohen Sulfataschegehalt den weiteren Verarbeitungsprozess stört. Die gemessenen Verschmutzungswerte können zur Planung von entsprechenden verfahrenstechnischen Anlagen zur Schmutzentfernung (Separatoren, Dekanter, Filter) herangezogen werden. Bei Einsatz von 3-Phasen-Dekantern könnte neben dem Schmutz- auch der Wassergehalt gesenkt werden.

Die schwankenden und hohen Werte der freien Fettsäuren bereiten bei der Umesterung gewisse Schwierigkeiten. Bei einem FFA-Gehalt von über 5 Mass.-\% müssen die freien Fettsäuren auf jeden Fall (sauer katalysiert) verestert werden bevor man in die basisch katalysierte Umesterung gehen kann.

Die relativ konstanten Werte bei den Viskositäten erleichtern die Auslegungen von Pumpen und Verrohrungen. 
In Bezug auf die spurenanalytischen Messergebnisse ist festzustellen, dass die Konzentrationen in den hier untersuchten Rohfettproben im Verlauf des Jahres 2001 relativ konstant waren und keine vereinzelt auffällig erhöhten Werte ermittelt wurden. Die Elementgehalte sowie die Gehalte an den anorganischen und organischen Schadstoffen sind sicherlich als Grundbelastung dieses Rohfettes anzusehen. Im Falle der Feststellung deutlich höherer Schadstoffkonzentrationen wäre von einem unbeabsichtigten bis illegalen Fremdeintrag auszugehen. Diese Möglichkeit sollte in jedem Fall nicht außer Acht gelassen werden, wie die zahlreichen Futtermittelskandale in der Vergangenheit belegen.

Nach der hier vorgestellten Bestimmung von Eigenschaften und Qualität des Rohstoffs, technische tierische Fette, ist nun u.a. beabsichtigt, den technischen Prozess zur Herstellung der Kühlschmierstoff-Grundöle, der aus mehrfacher Umesterung und Reinigungs- bzw, Fraktionierungsschritten besteht, analytisch zu begleiten. Es soll geklärt werden, ob hierbei Schadstoffe neu gebildet werden und in welchen Prozessfraktionen sich die o.g. Schadstoffe beim Vorliegen einer Kontamination der Rohstoffe anreichern würden.

\section{Literatur}

[1] Meyer-Pittroff R, Stammel V (2001): Thermische Verwertung von Produkten der Tierkörperbeseitigungsanlagen. VDI-Berichte Nr. 1622, ISBN 3-18-091622-2

[2] Deutsche Gesellschaft für Fettwissenschaft e. V. (2001): Deutsche Einheitsmethoden zur.Untersuchung von Fetten, Fett- produkten, Tensiden und verwandten Stoffen. C-III 17 a: Bestimmung von PAK in Ölen und Fetten. Wissenschaftliche Verlagsgesellschaft mbH, Stuttgart, ISBN 3-8047-1845-0

[3] Amtliche Sammlung von Untersuchungsverfahren nach $\$ 35$ LMBG: Verfahren zur Probenahme und Untersuchung von Lebensmitteln, Tabakerzeugnissen, kosmetischen Mitteln und Bedarfsgegenständen. 40 (1980 ff.): Bestimmung von Benzo[a]pyren in (geräucherten) Fleischerzeugnissen. Beuth Verlag, Berlin; ISBN 3-410-11220-0

[4] Nottrodt A (2001): Technische Anforderungen und allgeméine Empfehlungen für die Entsorgung von Tiermehl und Tierfett in Verbrennungsanlagen. Erstellt im Auftrag des Bundesministeriums für Umwelt, Naturschutz und Reaktorsicherheit

[5] Deutsche Gesellschaft für Fettwissenschaft (2001): Pressemitteilung: PAK-Verunreinigungen in Speiseölen. www.dgfert. de/press/pak.htm

[6] Vemmer H, Heeschen W, Blüthgen A, Daenicke R (1993): Übergang polychlorierter Biphenyle (PCB) aus Futtermitteln in Gewebe von Mastbullen. Landbauforschung Völkenrode 43, Heft 1, 27-33

[7] Bundesgesetzglatt (1998): Verordnung über Höchstmengen an Schadstoffen in Lebensmitteln, vom 23. März 1988. Bundesgesetzblatt: Verordnung zur Änderung der Schadstoff-Höchstmengenverordnung, vom 3. März 1997

[8] Verordnung zum Schurz vor gefährlichen Stoffen (GefStoffV), Neufassung vom 15.11.1999 (BGBl. 1 S. 2233), zuletzt geändert am 26.06.2000 (BGBl. 1 S. 932)

Eingegangen: 21. November 2002 Akzeptiert: 7. März 2003 OnlineFirst: 10. März 2003

\section{Deutsche Gesellschaft für Fettwissenschaft (http://www.dgfett.de)}

Geschichte. Die Deutsche Gesellschaft für Fettforschung wurde am 6.10.1936 gegründet und als gemeinnützige Gesellschaft in das Vereinsregister Berlin eingetragen. Gründungspräsident war Prof. Dr. Hans Paul Kaufmann.

Nach dem Krieg verlegte sie ihren Sitz nach Münster und wurde am 19.8.1948 als Deutsche Gesellschaft für Fettwissenschaft (DGF) wiedergegründet. Bereits 1949 konnte in Münster die erste Nachkriegstagung stattfinden.

Auf Anregung der DGF wurde am 26.10.1954 in Hannover die International Society for Fat Research gegründet, der heute 24 nationale Vereinigungen angehören.

Die DGF war langjährige Trägerin des Instituts für industrielle Fettforschung, das am 1.1.1969 als »Institut für Biochemie und Technologie der Fette - H.P. Kaufmann-Institut « in die seit 1964 bestehende Bundesanstalt für Fettforschung eingebunden wurde. Damit wurde die Bundesanstalt in ein "Institut für allgemeine und analytische Chemie « und in das genannte ehemalige DGF-Institut gegliedert.

Am 1.1.1996 wurde die Geschäftsstelle der DGF von Münster nach Frankfurt am Main verlegt. Danach begann eine Phase der Inter- nationalisierung der Kongresse und der Publikationen, die mit der Gründung der European Federation for the Science and Technology of Lipids am 9.10.2000 in Würzburg einen vorläufigen Höhepunkt erreicht hat.

Aufgaben der DGF. Die Gesellschaft verfolgt das Ziel, auf dem Gebiet der Fettwissenschaft und -technologie Fachleute aus Wissenschaft, Technik und Wirtschaft zu einer Gemeinschaftsarbeit zu vereinigen und ihnen ein Forum für den Gedankenaustausch zu bieten, wissenschaftliche und anwendungsorientierte Forschungsarbeiten zu fördern und ggf. eigene Forschungseinrichtungen zu unterhalten, die fachliche Ausbildung zu fördern, der Öffentlichkeit unabhängige Expertisen zu bieten und als Informationsquelle zu Fragen der Fettwissenschaft und -technologie zu dienen, Normen vorzubereiten, zu prüfen und Einheitsmethoden auszuarbeiten, hervorragende wissenschaftliche Arbeiten und Forscherpersönlichkeiten auszuzeichnen. Dadurch fördert die Gesellschaft eine umfassende interdisziplinäre Zusammenarbeit der naturwissenschaftlichen, medizinischen, technologischen, landwirtschaftlichen und ökologischen Forschung über Fette und Öle, Fettprodukte und -begleitstoffe, Tenside sowie Rohstoffe. 